\title{
GESTIÓN DEL PROCESO DE EXTENSIÓN UNIVERSITARIA PARA LA FORMACIÓN DE UNA CULTURA DE SALUD
}

\section{Management of university extension process for the formation of a culture of bealth}

\author{
Dagneris Batista de los Ríos \\ Universidad de las Tunas. Cuba \\ Correo-e: dagnerisbr@ult.edu.cu \\ C. Yanet Trujillo Baldoquin \\ Universidad de las Tunas. Cuba \\ Correo-e: yanettb@ult.edu.cu \\ C. Yoenia Barbán SARduY \\ Universidad de las Tunas. Cuba \\ Correo:ybarbán@ult.edu.cu \\ Recepción: 2 de abril de 2016 \\ Envío a informantes: 6 de abril de 2016 \\ Aceptación definitiva: 2 de mayo de 2016
}

Resumen: En la Universidad de las Tunas, a partir de la gestión del proceso de extensión universitaria, se trabaja por consolidar la relación con las comunidades, promover la cultura de salud en la sociedad en correspondencia con sus necesidades, favorecer una mayor calidad de vida del individuo y del medio que lo rodea, promover hábitos de vida saludables, conductas sexuales responsables, fortalecer el trabajo educativo y la formación de valores, mediante los estudiantes que se forman como promotores de salud, los cuales desde una perspectiva sociocultural logran una transformación positiva y un impacto en la comunidad universitaria y del contexto.

PALABRAS Clave: gestión; extensión universitaria; promotor; prevención.

Aвstract: At the University of Las Tunas from the management of university extension process, working to consolidate the relationship with communities, promote the culture of health in society in accordance with their needs, to promote a higher quality of life of the individual and the surrounding environment, promote healthy lifestyles, responsible sexual behavior, strengthen the educational work and the formation of values by students who are trained as health promoters, which from a sociocultural 
perspective achieve a positive transformation and impact in the university community and context.

KEY WORDS: management; university extension; promoter; prevention.

\section{Introducción}

$\mathrm{H}$

OY LA EDUCACIÓN SUPERIOR CUBANA ha ganado mayor pertinencia, cada día se abren aún más las puertas de sus instituciones que han salido de sus predios más tradicionales, no para fragmentarse, sino para ampliar su propio concepto de institución de Educación Superior y multiplicarse en toda la sociedad, conformadas por una sede central y varias sedes municipales, que de manera orgánica tributan entre sí y se enriquecen mutuamente.

En las universidades como tarea esencial está la formación integral de los estudiantes, desde una cultura académica, científica y laboral, para lograrlo se debe dar cumplimiento a acciones en las estrategias y programas nacionales de promoción y prevención, para elevar la cultura de salud de forma individual y colectiva. En la función de extensión niversitaria, con la creación y funcionamiento de formas organizativas diversas, se protege mejor la diversidad cultural existente propiciando un ambiente de desarrollo de una cultura general integral, favoreciendo el bienestar de las comunidades y elevando su calidad de vida.

Lo más relevante es como a partir de una correcta planificación las acciones extensionistas diseñadas no se muestran aisladas, sino que se complementan de manera que una acción le dé continuidad a otra y así sucesivamente, como lo es la formación de gestores o promotores de salud, a través de asignaturas facultativas o cursos, que estos reciben, lo que los dota de conocimientos que estos aprenden y luego ponen la práctica.

Los estudiantes aprenden cómo abordar desde el punto de vista sociológico y sociocultural las estrategias y programas de salud que se ejecutan en nuestras universidades, conocer el establecimiento de las relaciones existentes entre las condiciones y estilos de vida, factores y conductas de riesgo, la relación del hombre con su entorno social aportando elementos de importancia para el trabajo de promoción y de prevención en la comunidad, priorizando la concepción actual de los valores y ampliando el nivel de conocimientos de los individuos, utilizando como instrumento fundamental las manifestaciones artísticas a través de las cuales se puede tributar al tema desde una nueva perspectiva en aras de elevar la cultura de salud que es un elemento insoslayable de la formación integral.

\section{Desarrollo}

La educación superior debe ir a la vanguardia de todo combate para sembrar las ideas de que solo con mayor equidad y justicia podremos salvar al mundo en su diversidad biológica y cultural y construir sociedades donde el ser humano sea el centro de atención.

La salud constituye para el Estado cubano una prioridad gubernamental y social, ya que se considera como parte consustancial de desarrollo vinculada a todos los 
aspectos de la vida humana y constituye una de las prioridades dentro de las estrategias de los programas de calidad de vida que se ejecutan en nuestras universidades.

La Universidad de Las Tunas ha asumido un rol activo en la formación de una cultura de salud desde el enfoque educacional y cultural, potenciando acciones con un amplio espectro creativo desde el arte y la literatura en sus diversas expresiones.

La universidad debe continuar desempeñando su papel protagónico en la investigación científica que se realiza en el país, en el desarrollo de tecnologías, participar en la innovación para satisfacer las demandas y necesidades de la sociedad. Es por ello que corresponde una constante generación y divulgación del conocimiento, hacer que desde el enfoque educacional y cultural se potencien acciones con un amplio espectro creativo desde el arte y la literatura en sus diversas expresiones, para una cultura de salud que dé respuesta a las necesidades e intereses de la comunidad universitaria y a la del contexto.

En la Primera Conferencia Latinoamericana de Extensión Universitaria y Difusión Cultural (1957), se expresó: «Por su naturaleza la Extensión Universitaria es misión y función orientadora de la universidad contemporánea, entendida como ejercicio de la vocación universitaria. Por su contenido y procedimiento se funda en estudios y actividades filosóficas, científicas, artísticas y técnicas. Por su finalidad debe proyectar dinámica y coordinadamente la cultura y vincular al pueblo con la universidad, estimular el desarrollo, elevar el nivel espiritual, intelectual y técnico del pueblo». Este criterio sintetiza cuál es el objetivo que debe tener el proceso de extensión universitaria desde una concepción pedagógica, en el que se establece la relación de los contenidos y los procedimientos de su gestión, para lograr una formación integral en los estudiantes.

Álvarez de Zayas (1996a: 47) refiere que «el estudiante aprende, estudia, en tanto resuelve un problema, en tanto se estimula, porque como resultado de su labor es más inteligente, y en el contexto social, está más cerca de los valores que la sociedad establece como modelo». Este autor reconoce que «en el contenido se sintetiza una parte de la cultura que se selecciona con criterios pedagógicos con el propósito de formar integralmente al educando». El contenido desempeña un rol importante en la formación del futuro profesional; posee un carácter activo, capaz de transformarse, relacionado con la esencia de la cultura, ya que el contenido incluye el sistema de conocimientos como reflejo del objeto de estudio y el sistema de habilidades que encierra el conjunto de relaciones del hombre con ese objeto y de los hombres entre sí: relaciones sociales y que expresan la riqueza, la diversidad y la naturaleza multidimensional del proceso.

Por eso, y a tenor de los fundamentos precedentes, la gestión de la extensión universitaria desde el punto de vista sociológico se presenta de manera multidimensional donde se atienden todos los procesos formativos y aprovecha las potencialidades de los contenidos que se aportan en cada uno de ellos. La cultura es un componente fundamental, ya que se convierte en un instrumento para estimular el cambio de conducta y modificar actitudes. Las manifestaciones artísticas (música, teatro, danza, literatura, artes plásticas, cine), el deporte, de conjunto con los medios de divulgación, no se deben utilizar solo para la recreación, sino a su vez para educar a las personas, para motivarlas a participar en debates, estimular un cambio de conducta, aumentar la tolerancia e influir en los observadores para que intercambien la información que han conocido por otras personas, promocionar hábitos de vida saludables, conductas sexuales responsables, en fin, producir un ciclo de conocimientos. 
Esta investigación tiene sus inicios en el curso 2005-2006, y después de una década se aprecian resultados palpables y un impacto en la formación y trabajo de los estudiantes formados como promotores de salud, que de diferentes años y carreras se han involucrado en proyectos comunitarios y hoy constituye un movimiento dinamizador en la universidad que penetra con más eficacia en los diferentes grupos sociales a través de la educación de pares o iguales.

La educación y asesoramiento de jóvenes por otros jóvenes ha pasado a ser uno de los enfoques más comunes para abordar la salud en sus diferentes temáticas, por lo que su intervención en la prevención y educación ha ganado terreno y aceptación en los últimos años. En el proceso de dispensarización que se realiza al iniciar cada curso escolar en cada una de las brigadas de estudiantes se identifican los grupos de riesgo, con los que se trazan acciones medibles y personalizadas, en este proceso aunque intervienen profesores guías, instructores educativos, tutores y otros agentes educativos, es vital el trabajo de los promotores para lograr cambios de conducta y comportamientos con patrones de fácil reconocimiento para los estudiantes y extender el trabajo de promoción y de prevención a toda la comunidad universitaria y a la comunidad del entorno.

Debemos destacar que, cuando hablamos de promotores de la salud, están presentes estudiantes de carreras humanísticas, técnicas, económicas y agrícolas, por lo que el espectro es mucho mayor. La participación de estudiantes como promotores de salud en grupos sociales inspira sentimientos de conexión y pertinencia, los ayuda a desarrollar un sentido de identidad y fortalece sus ideas y valores. A partir del Manual Básico para las Universidades Cubanas para la formación de promotores en la prevención del viH/sida, la Dirección de Extensión Universitaria de la Universidad de Las Tunas diseñó el programa analítico de un curso facultativos para la formación de promotores de la salud, adecuándolo a los intereses, objetivos, necesidades sentidas y reales de la comunidad a la cual se van a destinar las acciones de prevención.

El programa incluye las informaciones básicas de las ITS/viHsidA y la situación epidemiológica en la provincia, conocimientos del cuerpo humano, sexualidad, autoestima, familia, ética, actitudes de riesgo, prácticas sexuales, temas generales de las drogas, diversidad sexual, tolerancia, efectos negativos de la automedicación, instrumentos para abordar desde el punto de vista sociológico y sociocultural el trabajo de promoción y de prevención, relaciones entre las condiciones y estilos de vida, factores y conductas de riesgo, relación del hombre con su entorno social, trabajo de promoción y de prevención en comunidades, aspectos de la comunicación, técnicas participativas, temas de cultura relacionados con las manifestaciones artísticas.

El proceso de formación de promotores de salud no es masivo, es selectivo, ya que no todos poseen aptitudes para motivar, explicar, convencer o realizar actividades de promoción, por lo que esto presuponía una barrera. Por todo lo anteriormente expuesto, surgía una interrogante: ¿qué hacer con aquellos estudiantes que estaban interesados en formarse como promotores de la salud y no poseían estas habilidades o con aquellos que formaban parte dentro de la comunidad como líderes y no se sentían motivados?

Los objetivos del curso en cuanto a estructura, conocimientos y actitudes previstas a desarrollar se cumplían, pero era necesario rediseñar el mismo buscando incluir elementos de interés que motivaran a nuestros estudiantes. Se aplicaron a los estudiantes variables de medición para conocer actitudes personales, aspiraciones, 
motivaciones, prejuicios, capacidad para la comunicación, gustos, tabúes, creencias, necesidades sentidas e identificación de los valores. A partir de las respuestas obtenidas, se rediseñó el curso, ya no sería una actividad meramente docente, sino un espacio que motivaría la búsqueda de información, propiciaría la discusión y reflexión constante, para ampliar sus potencialidades artísticas y elevar de forma general su cultura sexual y su cultura general integral.

Existieron coincidencias en muchos aspectos, pero lo que más nos llamó la atención fue la inclinación a las manifestaciones artísticas, especialmente música, cine, artes plásticas y literatura, a pesar de no tener hábitos de lectura en la gran mayoría. Se decidió vincular los contenidos con las manifestaciones artísticas, con el objetivo de que a partir del análisis reflexivo individual se aportaran elementos que propiciaran tributar al tema desde una nueva perspectiva, enriquecer el trabajo de promoción y enriquecer así la cultura general integral de la comunidad.

En el curso se incluyeron elementos de todas las manifestaciones, pero se le prestó especial atención a la literatura. Uno de los mayores desafíos que se tienen en la formación de futuros profesionales es desarrollar sus capacidades lectoras y que descubran las potencialidades que encierra la lectura en la construcción de la subjetividad para que puedan dimensionar el valor que tiene en su formación.

Partimos del concepto de que la lectura y la escritura no son solo una vía de desarrollo del conocimiento y de los procesos cognitivos, sino también un camino para contribuir a construir la subjetividad, la identidad, fortalece los valores existentes y aporta nuevos elementos a nuestro vocabulario para poder comunicarnos. Es necesario conocer que la subjetividad no es más que la capacidad del hombre de plantearse a sí mismo como sujeto, solo es posible por la conciencia de sí mismo, por eso tiene una propiedad reflexiva, es el reconocimiento de los estados mentales de uno mismo y de los otros. Para construirla se debe tener su propio punto de vista y su propia visión. En cambio los valores están arraigados en la misma condición de la existencia, constituyen un punto de mira y el objetivo último en la formación de toda la personalidad, permiten construir la base para establecer la propia identidad y un modo personal de relacionarse con el mundo. Son los principios-guías que orientan y comparten los miembros de una institución educativa.

El modo de vincularse que tienen los hombres es a través del lenguaje, por eso la unidad básica de interacción social son los textos-discursos. Es en la comunicación humana donde los individuos se representan a sí mismos y al mundo, así como significan sus acciones y la realidad. El principio constitutivo del lenguaje es el diálogo, sin embargo, no debe reducirse a la comunicación interpersonal entre un locutor y su interlocutor, sino que presupone relaciones con otros enunciados.

A este entrecruzamiento de voces Bajtin lo denomina polifonía, que no es más que la presencia de otras voces, puntos de vista, espacios mentales, posiciones sociales y perspectivas axiológicas que dejan ver las huellas o marcas del locutor en sus discursos. Esto se traduce en el trabajo de promoción y de prevención en las comunidades, donde se propicia un espacio para la reflexión, se intercambian puntos de vista, se promocionan hábitos de vida saludables, conductas sexuales responsables, todo en aras de elevar la calidad de vida de los individuos

La sociología concibe la lectura como habilidades que pueden ser enseñadas en cualquier contexto y en cualquier situación social, esta forma parte de las prácticas culturales, que se desarrolla y construye en las relaciones sociales, a través de 
la formación y de una necesidad creada por valores compartidos socialmente no solo como vía para obtener información, sino como una práctica para emplear el tiempo libre.

Para poder ser un buen promotor se necesita, independientemente del conocimiento básico y de técnicas que contribuyan al trabajo de promoción, un lenguaje que pueda ser fácilmente decodificado en la comunidad o en los grupos sociales donde se interactúa. El interés por la lectura no depende de una iniciativa individual, no se forma un lector a fuerza de voluntad, la voluntad que mueve al lector a interesarse por la lectura es una construcción social.

Partimos conceptualmente de que la lectura es transversal a todos los espacios de la vida social, en los ámbitos económicos, científicos, políticos, jurídico, medioambiental, de prevención y, por qué no, en el ámbito sexual, ya que esta alternativa les permite a todos y en este caso a los promotores enriquecer el vocabulario, no recurrir a frases hechas o rígidas, resaltando su propia voz, siendo auténticos, con sentido de pertenencia de lo que se expresa, con opiniones fundamentadas propias de estudiantes universitarios que se convertirán en futuros profesionales.

Las actividades donde se activarán los procesos cognitivos, no se abordaron como un conjunto de procesos formales en sí mismos, descontextualizados, ajenos a los estudiantes, sino a través del debate de libros y materiales que reflejaran de una manera diferente las temáticas relacionadas con la pandemia del viH/sIDA, la diversidad sexual, características de la sexualidad, entre otros tópicos. Se iniciaron los debates con varios libros que abordaban la temática, como por ejemplo cuentos del libro La noche al revés, del escritor tunero Ramiro Duarte, un libro donde se describen situaciones que no equidistan de la realidad con un lenguaje directo.

El recurso utilizado logró el efecto de motivar y despertar el interés; a partir de este momento se seleccionaron otros materiales que propiciaran la observación, la conceptualización de temáticas, la explicación de las causas que producen determinados hechos como lo son las actitudes de riesgo, la tolerancia, la baja percepción de riesgo, la necesidad de fomentar los valores de la familia, la responsabilidad, la honestidad, la fidelidad entre otros.

Con este trabajo se logró motivar la búsqueda de literatura especializada y no circunscribirse con lo que se recibía en el curso, propiciar el debate de obras relacionadas o no con el tema, promocionando hábitos de lectura. Este tema compromete en lo afectivo, en lo valorativo, en los prejuicios existentes, los tabúes, los valores, en las creencias, por lo que es necesario propiciar el debate para que sean ellos los que llegan a sus propias conclusiones y no sean impuestas, sino que las tomen a partir de la reflexión de acciones consecuentes.

Para conocer el impacto del trabajo de los promotores a partir del nuevo programa que se aplicaba se aplicaron variables de medición antes del curso y posterior a este. Resulta significativo cómo los intereses, necesidades y el bajo nivel de conocimientos de los mismos se suplían a través del trabajo sistemático en este curso, elevándose el rendimiento académico, sentido de pertinencia, su nivel de compromiso y responsabilidad ante las nuevas tareas. Lo más importante, desde nuestra perspectiva, es que se obtiene realizar el trabajo de prevención desde otras aristas que van encaminadas a suplir las necesidades, intereses y objetivos de la comunidad universitaria, identificándose como parte de esta población heterogénea y vulnerable. 
D. BATISTA DE LOS RÍOS, C. Y. TRUJILLO BALDOQUIN Y C. Y. BARBÁN SARDUY

La utilización de elementos de manifestaciones artísticas logra una interrelación directa con la comunidad a través de la comunicación que permite una retroalimentación, ampliando el espectro de comunicación vinculando el tema con otros que resultan de interés para la comunidad universitaria. A partir de esta experiencia se alcanzó concientizar a aquellos que forman parte de los grupos de riesgo con atención personalizada, propiciar cambios de conducta, de comportamiento, y estimular a otros estudiantes a interesarse por formarse como promotores de salud como una labor humana, sensible y de gran importancia en estos momentos en el enfrentamiento de la pandemia.

\section{Conclusiones}

A través de las manifestaciones de literatura y artes plásticas se contribuyó a ampliar el conocimiento de los promotores de salud, con énfasis en el trabajo con los valores tan necesarios en la formación de estudiantes como mejores seres humanos. Se amplió la cultura sexual de la comunidad universitaria y de forma vertical y horizontal se elevó con este programa la cultura general integral de todos. A partir del trabajo que se realizó en el centro con la formación de estudiantes como gestores o promotores de salud, se obtuvieron resultados de impacto en la comunidad universitaria, promoviendo una cultura de salud; se logró fomentar hábitos de lectura, ampliando el nivel de comunicación de los mismos; se fortalecieron los valores en los estudiantes, y se logró mediante la gestión de esta actividad extensionista visualizar el papel que la universidad actual puede llevar a cabo en la comunidad y en la sociedad.

\section{Bibliografía}

Acconcia, M. L. y col. (20I2) La extensión universitaria como espacio dialógico de construcción y problematización de saberes y prácticas. Registrado en: http://revistas.unc.edu.ar/ index.php/ext/article/view/1855.

Addine Fernández, F. (2006) Modo de actuación profesional pedagógica. De la teoría a la práctica (pp. 7-Io). La Habana: Editorial Academia.

Alarcón De Quesada, R. (2002) 40 aniversario de la Reforma Universitaria. La Habana.

Alarcón Ortiz, R. (20I5a) Conferencia inaugural en el XIII Congreso Latinoamericano de Extensión Universitaria. La Habana.

Alarcón Ortiz, R. (2015b) Las ciencias de la educación en una universidad integrada e innovadora. Pedagogía 2015. La Habana.

Alarcón Ortiz, R. (2015c) La Educación Superior en el sistema nacional de ciencia, tecnología e innovación. La Habana.

Alarcón Ortiz, R. (2016) Conferencia inaugural en el X Congreso Internacional de Educación Superior Universidad 20I6. La Habana.

Alfaro Pérez, J. (1997) La extensión y la acción social universitarias en Costa Rica. Revista Imágenes, vol. 4, n. $^{\circ}$ 7. Costa Rica.

Alfaro Pérez, J. y col. (1999) Integración de las áreas académicas: consolidación de un modelo universitario. Revista Imágenes, vol. 6, n. ${ }^{\circ}$ 9, I87-190. Costa Rica.

Álvarez de Zayas, C. M. (1990) Fundamentos teóricos de la dirección del proceso docente educativo en la Educación Superior Cubana. Ciudad de La Habana: Imprenta Andre Voisin. 
Álvarez de Zayas, C. M. (1996a) La escuela en la vida. La Habana: Editorial Félix Varela.

Álvarez de Zayas, C. M. (1996b) Hacia una escuela de excelencia. Santiago de Cuba: Editorial Centro de Estudios Educación Superior Universidad de Oriente.

Álvarez de Zayas, R. M. (i995) La formación del profesor contemporáneo, currículum y sociedad. Pedagogía 95. La Habana.

Álvarez de Zayas, R. M. (1997) Hacia un currículo integral y contextualizado. La Habana: Editorial Academia.

Ander Egg, E. (1993) La planificación educativa. Conceptos, métodos, estrategias y técnicas para educadores. Argentina: Editorial Magisterio del Río de la Plata.

Aponte, C. y col. Propuesta de indicadores de evaluación de la función de proyección social/ extensión universitarialinteracción en la educación superior. Documento de trabajo ASCUN. Colombia.

Araujo da Silva, A. G. (20I5) As politicas de educação (em tempo) integral e a tecnología empresarial da gestão por resultados. Brasil: Universidade Federal de Alagoas. Publicado en Pedagogía 20I5.

Artiles Espinosa, S.; Ledón Mora, M. y Sosa Sosa, B. E. (20io) Integración de agencias y agentes socializadores, escuela, familia, otras instituciones de la comunidad, organizaciones políticas y de masas. Revista Contribuciones a las Ciencias Sociales. Registrado en: www. eumed.net/rev/cccss/ro/.

Ballesteros, M. V. (2010) La Extensión Universitaria como proceso de Comunicación. Revista Electrónica de Extensión Universitaria, n. ${ }^{\circ}$ 2. Argentina.

BATISTA DE LOs Ríos, D. (20Io) Orientación educativa en la universidad actual desde la dimensión extensionista. Revista Conexão UEPG, vol. 6 , n. ${ }^{\circ}$ I. Portugal.

BATISTA DE los Ríos, D. (2OI2a) Compromiso social de las universidades en el fortalecimiento de la identidad cultural a través de la memoria viva de la comunidad.Revista Didasc@lia: Didáctica y Educación, vol. 3 , n. ${ }^{\circ}$. Las Tunas.

Batista De los Ríos, D. (20I2b) Liderazgo de la universidad en la sociedad del conocimiento desde la dimensión extensionista. Revista Electrónica de Extensión Universitaria, n. ${ }^{\circ}$ 3. Argentina. Registrado en: www.perio.unlp.edu.ar/extensionenred.

Batista de los Ríos, D. y Sánchez Bustos, C. (20I3) Evolución histórica de la Universidad de Las Tunas desde la perspectiva extensionista. Revista Iberoamericana de la Educación Superior, vol. 4, n. ${ }^{\circ}$ 9. Brasil.

Batista Gutiérrez, T. (2007) La gestión pedagógica en el año académico desde un modelo integrador del currículo y la labor educativa. Revista Institucional Universidad Tecnológica del Chocó, n. ${ }^{\circ} 26$.

Bermúdez Morris, R. y Pérez Martín, L. M. (2004) Aprendizaje formativo y crecimiento personal. La Habana: Editorial Pueblo y Educación.

Botero Chica, C. A. (2009) Cinco tendencias de la gestión educativa. Revista Iberoamericana de Educación, n. ${ }^{\circ} 49 / 2$.

OchOA, R. y col. (2006) Trabajo multisectorial en VIH/SIDA. Manual Práctico metodológico.

Veliz Gutiérrez, J. A. y col. (20II) La Extensión Universitaria y la Promoción de Salud en la Atención Primaria en la Universidad Médica de Pinar del Río. Revista de Ciencias Médicas, vol. I5, n. ${ }^{\circ}$ 4. Pinar del Río. Registrado en: http://www.revcmpinar.sld.cu/index.php/publicaciones/article/view/848.

Vento Tielves, R.; Pimentel Rivero, I.; Villarreal Domínguez, J. y Valdés Corrales, R. J. El papel de la Extensión Universitaria en la formación del profesional de la carrera de Agronomía de Montaña de San Andrés. Pinar del Río. Soporte digital.

Vezub Lea, F. (2007) La formación y el desarrollo profesional docente frente a los nuevos desafíos de la escolaridad. Revista de Currículum y Formación del Profesorado, n. ${ }^{\circ}$ II.

Vigostri Lev, S. (1987a) Pensamiento y lenguaje. La Habana: Editorial Pueblo y Educación.

Vigostki Lev, S. (I987b) Historia del desarrollo de las funciones psíquicas superiores. La Habana: Soporte Digital. 